\title{
A Successfully Treated STEMI Due to Simultaneous Thrombotic Occlusion of Left Anterior Descending Artery and Left Circumflex Artery: A Case Report and Review of the Literature
}

\author{
Michal Kuzemczak a, b, c, f, Ryszard Kasinowskib, Piotr Skrobich ${ }^{\mathrm{d}}$, \\ Roland Podlewski ${ }^{a}$, Piotr Kalmucki ${ }^{b}$, e
}

\begin{abstract}
ST-segment elevation myocardial infarction (STEMI) due to simultaneous double vessel thrombotic occlusion of two major coronary arteries is an extremely rare clinical entity. Available studies indicate that most frequently it affects two coronary arteries originating from different sides of a coronary tree (i.e. right coronary artery (RCA) and left anterior descending artery (LAD) or RCA and left circumflex artery (LCx)) and usually has a fatal clinical course. However, it must be pointed out that the data have been derived from studies before the era of a widely-used pre-hospital electrocardiogram (ECG) teletransmission. Herein, we present a case report of successfully-treated STEMI due to simultaneous occlusion of LAD and LCx. Furhtermore, the case report highlights a crucial role of ECG teletransmission for immediate treatment and survival of patients with the so-called "the deadly double infarct syndrome".
\end{abstract}

Keywords: Multivessel STEMI; Multiple culprits; ECG teletransmission

\section{Introduction}

ST-segment elevation myocardial infarction (STEMI) is a clin-

Manuscript submitted October 26, 2018, accepted November 12, 2018

${ }^{a}$ Department of Medical Rescue, University of Medical Sciences, Poznan, Poland

b Department of Intervetional Cardiology, Cardiac Hospital, Kowanowko, Poland

${ }^{c}$ CTVS/Myocardial Restoration Laboratory, Department of Surgery, National University of Singapore, Yong Loo Lin School of Medicine, Singapore dDepartment of Cardiology, Cardiac Hospital, Kowanowko, Poland eHCP Medical Center, Department of Interventional Cardiology, University of Medical Sciences, Poznan, Poland

${ }^{\mathrm{f}}$ Corresponding Author: Michal Kuzemczak, CTVS/Myocardial Restoration Laboratory, Department of Surgery, Yong Loo Lin School of Medicine, National University of Singapore, MD6 Building, Level 8 South, 14 Medical Drive, Singapore 117599, Singapore. Email: michal.kuzemczak@gmail.com

doi: https://doi.org/10.14740/cr798 ical entity being most commonly a consequence of unstable coronary plaque rupture or erosion leading to platelets activation, thrombus formation and occlusion of an infarct-related artery (IRA) [1]. In vast majority of cases, there is a single culprit lesion in a coronary tree. However, although very uncommon, STEMI due to simultaneous double coronary thrombosis has been reported [2]. Because of high mortality rates and poor prognosis, it has been called "the deadly double infarct syndrome" $[2,3]$. Therefore, early diagnosis and emergent percutaneous coronary intervention (PCI) is crucial for the patients' survival.

Available data indicate that multiple culprit lesions most commonly affect coronary arteries originating from different sides of a coronary tree, i.e. simultaneous occlusion of right coronary artery (RCA) and left anterior descending artery $(\mathrm{LAD}) /$ left circumflex artery $(\mathrm{LCx})$ is observed [2]. Herein, we present a case report of a rare presentation of STEMI due to simultaneous double thrombotic occlusion of two coronary arteries originating from the same side of a coronary tree (i.e. LAD and LCx) successfully treated with primary PCI. The case report highlights the importance of electrocardiogram (ECG) teletransmission for early diagnosis and survival of patients with this commonly fatal condition. Furthermore, a review of the available literature concerning double-vessel STEMI was performed.

\section{Case Report}

A 47-year-old obese male (height: $184 \mathrm{~cm}$, weight: $138 \mathrm{~kg}$, body mass index (BMI): 40.76) was admitted to our department of cardiology due to sudden onset of typical crushing retrosternal chest pain that had occurred during gardening. He had a history of hypercholesterolemia and stable coronary artery disesase. However, till that day, he had not undergone coronary angiography. Five years before admission, he had quited smoking cigarettes. Earlier, he had been a heavy smoker (20 cigarettes/day for 27 years). Positive family history was documented - patient's father had died due to sudden cardiac death (SCD). On admission, no other risk factor was documented.

The patient called emergency 20 min after the onset of retrosternal pain. Immediatelly after paramedics' arrival, the pa- 


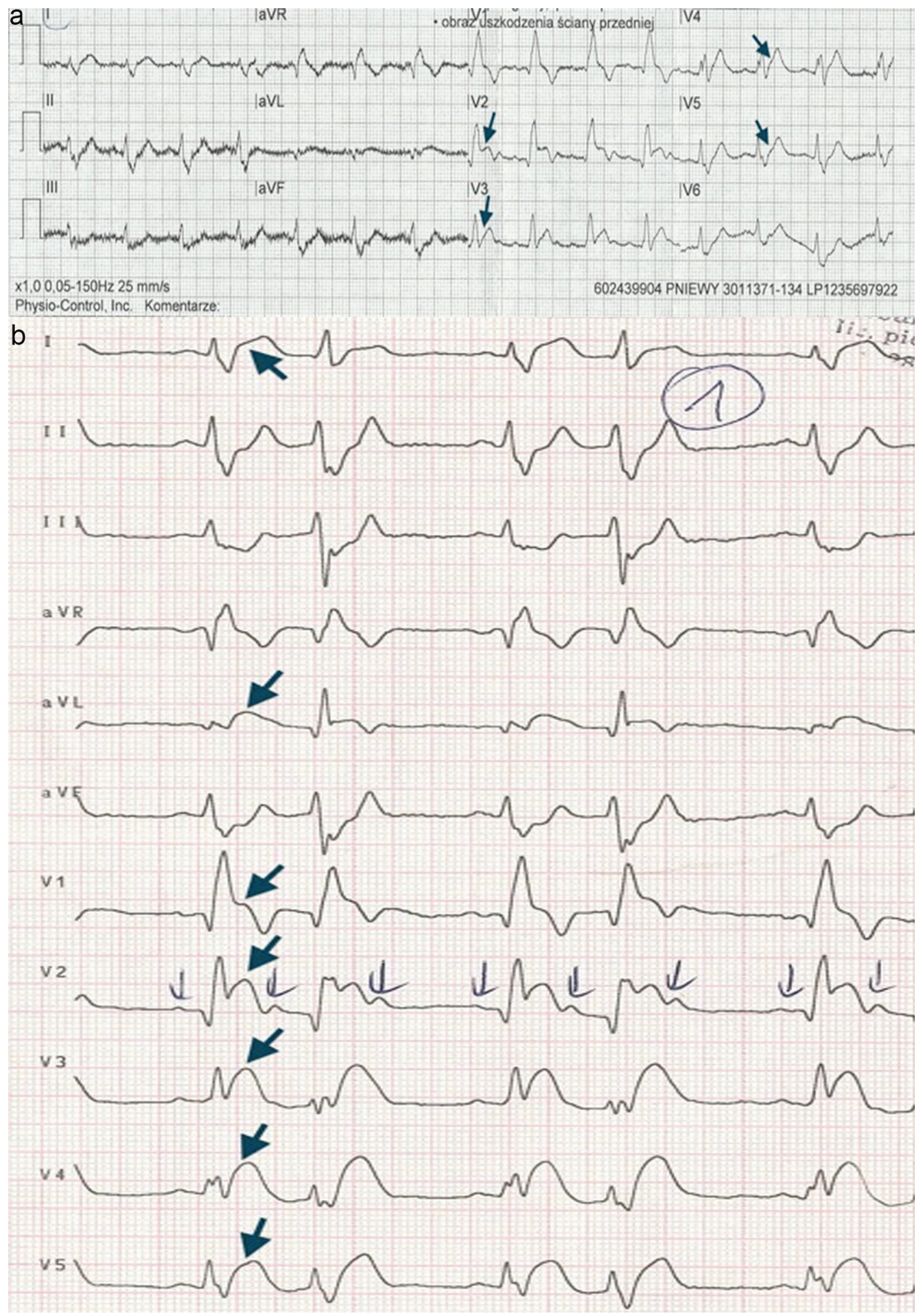

Figure 1. The patient's ECG. (a) ECG transmitted to the cath lab using LifeNet system (Physio Control, USA). ST-segment elevation in precordial leads V2 - V5 (black arrows). (b) ECG obtained immediately after arrival to the hospital. ST-segment elevation in precordial leads V1 - V6 and leads I, aVL (black arrows).

tient's ECG was transmitted to our department using LifeNet system (Physio Control, USA). The ECG revealed ST-segment elevation in precordial leads V2 - V5 (Fig. 1a). However, after arrival to the hospital, ST-segment elevation became more pro- nounced and diffuse. This ECG pattern was noticed in precordial leads V1 - V6 and leads I, aVL, which was consistent with anterolateral STEMI (Fig. 1b). Prior to arrival to the cath lab, the patient was preloaded with dual antiplatelet therapy (300 


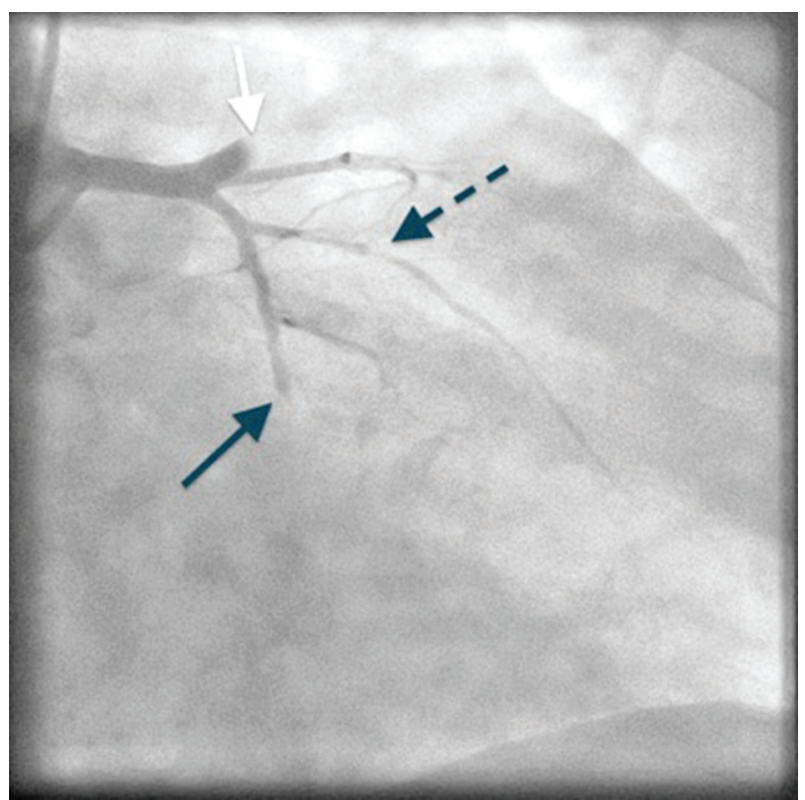

Figure 2. Coronary angiography of left coronary artery - a simultaneous occlusion of LAD (white arrow) and LCx (black arrow), a critical stenosis of first marginal branch (black arrow with interrupted line). LAD: left anterior descending artery; LCx: left circumflex artery.

$\mathrm{mg}$ acetylsalicylic acid (ASA) and $600 \mathrm{mg}$ clopidogrel orally) as well as 5,000 IU of unfractionated heparin (UFH) was intravenously administered. Furthermore, morphine was administered in order to relief chest pain.

On admission ( $1 \mathrm{~h}$ from the onset of symptoms), the patient was hemodynamically stable, but still suffered from retrosternal crushing pain. On examination, his heart rate was $110 / \mathrm{min}$, his blood pressure was $100 / 75 \mathrm{~mm} \mathrm{Hg}$ and his respiratory rate was 22/min with $\mathrm{SpO}_{2} 98 \%$ on room air. Physical examination did not reveal any significant abnormalities. On transthoracic echocardiography, akinesis of the apex, apical and medial segments of anterior wall as well as interventricular septum with ejection fraction of $44 \%$ were revealed. The patient's admission high-sensitivity cardiac troponin I level was slightly elevated at $20.3 \mathrm{ng} / \mathrm{L}$ (normal range: $<19 \mathrm{ng} / \mathrm{L}$ ), but it peaked in subsequent blood tests above $40,000 \mathrm{ng} / \mathrm{L}$.

The patient was taken to a cath lab equipped with a biplane digital angiography system (Siemens Axiom Artis; Siemens Inc., Germany) for emergent coronary angiography. The procedure was performed using right transradial approach. The patient's coronary angiography demonstrated total occlusion (TIMI 0 flow) by a fresh thrombus to both proximal segment of LAD (the sixth coronary segment) and mid-segment of $\mathrm{LCx}$, distally to first marginal branch (the thirteenth coronary segment) (Fig. 2). Furthermore, a critical stenosis in the first marginal branch of LCx was demonstrated. Right coronary angiography revealed a significant stenosis in its mid-portion (Fig. 3). Based on these findings, PCI in the setting of STEMI was performed immediately following coronary angiography. After intubation of left main stem by a $6 \mathrm{~F}$ extra backup (EBU) 3.75 guiding catheter (Launcher, Medtronic, USA), both occlusions were crossed using Whisper MS (Abbott Vascular, USA)

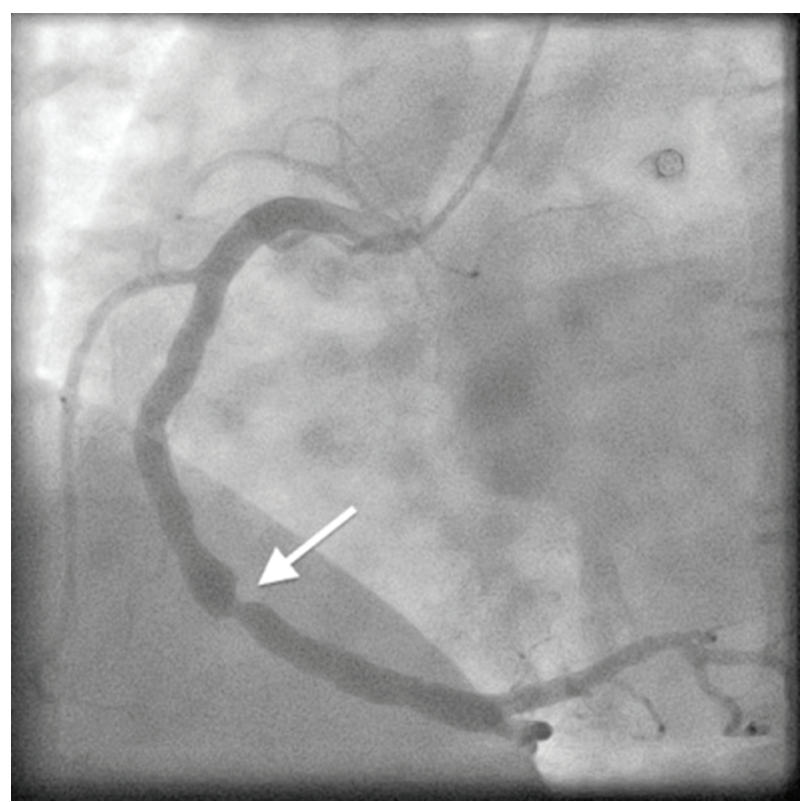

Figure 3. Coronary angiography of RCA - a significant stenosis in its mid-portion (white arrow). RCA: right coronary artery.

guidewires. Due to high thrombus burden in LAD, manual aspiration thrombectomy was performed with Aspicath (Biometrix, The Netherlands) thrombus aspiration catheter, resulting in a patent coronary artery with the presence of critical stenosis. After predilatation (14 atm) using a 3.0/20 mm Sapphire (OrbusNeich, Hong Kong, China) balloon dilatation catheter, $3.5 / 30 \mathrm{~mm}$ DES Xience Pro (Abbott Vascular, USA) was implanted $(16 \mathrm{~atm})$. The stent was postdilatated $(12-20 \mathrm{~atm})$ in its proximal and mid-part with a $3.75 / 20 \mathrm{~mm}$ non-compliant balloon NC Solarice (Medtronic, USA) with excellent angiographic result and normal coronary blood flow (TIMI 3 flow). Subsequently, PCI of the second IRA was performed. After predilatation (14 atm) using a 2.0/20 mm Sapphire (OrbusNeich, Hong Kong, China) balloon dilatation catheter, 2.5/28 $\mathrm{mm}$ DES Xience Pro (Abbott Vascular, USA) was implanted (10 - $12 \mathrm{~atm})$. PCI of both IRAs resulted in restoration of coronary blood flow (TIMI 3 flow in both IRAs) (Fig. 4). Gradual resolution of retrosternal pain as well as ST-segment elevation was observed. The patient was discharged a week later after uneventful hospital stay with scheduled elective PCIs of remaining significant but stable coronary lesions.

\section{Discussion}

In vast majority of cases, STEMI results from atheromatous plaque rupture or erosion with a subsequent thrombotic occlusion of one of the coronary arteries. Available data indicate that it is extremely rare in clinical practice to diagnose STEMI being a consequence of simultaneous total thrombotic occlusion of more than one major coronary artery [2]. These patients have a very poor prognosis and usually do not survive long enough to undergo coronary angiography. They die suddenly before arriving to a hospital or, in case of a more positive sce- 


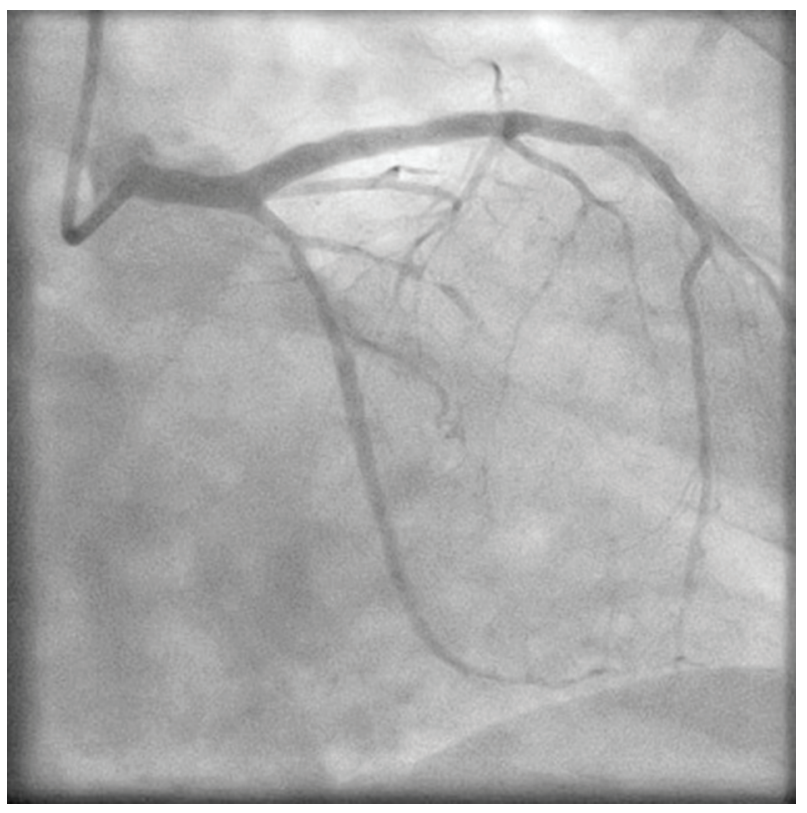

Figure 4. Coronary angiography of left coronary artery following PCls of both infarct-related arteries (LAD and LCx). PCls: percutanous coronary interventions; LAD: left anterior descending artery; LCx: left circumflex artery.

nario, presented with cardiogenic shock and rapid fatal clinical course. In the study of Polakk et al, most of them were hemodynamically unstable on presentation, with more than one-third in cardiogenic shock [2]. Therefore, early diagnosis and emergent PCI is of paramount importance for their survival. ECG teletransmission may shorten the time in which these patients reach a cath lab leading to improvement of clinical outcomes in this unique population. The case report presents a successfully-treated patient with double-vessel STEMI associated with simultaneous total occlusion of LAD and $\mathrm{LCx}$, in whom percutaneous intervention had been proceeded by ECG teletransmission to the cath lab.

As mentioned above, in majority of cases, STEMI is due to acute occlusion of one major coronary artery. Other vessels have no lesions, stable lesions (either significant or not) or chronic total occlusions. Acute occlusions of more than one major coronary artery is seldom encountered in clinical practice. Available studies suggest that their prevalence is estimated on $2.5 \%$ [2]. However, it must be pointed out that due to selection bias associated with catastrophic outcomes, their real incidence is probably significantly underestimated.

The most frequent angiographic finding in patients with double-vessel STEMI is occlusion of two coronary arteries originating from different sides of a coronary tree. In the study of Polakk et al, in orignal series consisting of 18 patients, half of them presented simultaneous occlusion of RCA and LCx [2]. After adding 29 cases that had been previously reported, the most frequent angiographic finding in total case series of 47 patients was occlusion of RCA and LAD. STEMI resulting from concomitant occlusion of LAD and LCx is a much rarer phenomenon. Fukaya et al reported a successfuly treated STEMI due to simultaneous occlusion of LAD and LCx [4].
However, in contrast to our case report, the patient was hemodynamically unstable and required intra-aortic baloon pump (IABP). It is intriguing that in our case, despite simultaneous occlusion of LAD and LCX, as well as significant stenosis of RCA, the patient was hemodynamically stable and had a relatively benign course. Undoubtedly, the time factor could play a significant role. In this case, pre-shospital ECG teletransmission enabled rapid initiation of effective treatment, reduced the time from the onset of symptoms to the opening of occluded arteries and prevented clinical deterioration of the patient.

The underlying putative mechanism responsible for mutiple culprits has not been fully elucidated. One of the postulated mechanisms is a diffuse destabilization of coronary plaques as a consequence of an inflammatory response that is not restricted to a single culprit lesion. The concept called "pancoronaritis" is all the more truthful considering the fact that atherosclerosis is a more generalized chronic inflammatory disease with exacerbations resulting in acute coronary syndromes. Therefore, it cannot be ruled out that these exacerbations may develop in a multifocal pattern leading to rupture of more than one coronary plaque [1]. Noteworthy, this has been confirmed by several studies. Goldstein et al indicated that more than $30 \%$ of patients with acute myocardial infarction had multiple plaque ruptures with overlying thrombi [1]. In the study of Burke and Virmani, even half of patients who died due to acute myocardial infarction had multiple thromboses in their coronary tree on autopsy [5]. On the other hand, plaque raptures are also observed in patients with stable angina as well as asymptomatic patients [6]. Clinical consequences are an aftermath of interplay between numerous factors, i.e. severity of coronary thrombosis, vessel diameter, tonus of vasculature, adrenergic surge, etc. While many of them (factors intrinsic to coronary plaques as well as extrinsic forces) have been well described, some mechanisms contributing to plaque instability still have not been fully delineated. Furthermore, multiple plaque ruptures in the setting of STEMI can be not only the effect of simultaneous, but also sequential thrombotic occlusion of two major coronary arteries $[7,8]$. The latter is considered to be a consequence of hemodynamic instability and hypotension due to occlusion of one coronary artery, resulting in blood stasis and acute occlusion in another coronary artery with severe underlying lesion [2]. Therefore, some of the cases reported as simultaneous occlusions might have resulted from sequential occlusions within a short period of time [7]. Based on published case reports, among other factors predisposing to hypercoagulability and multivessel coronary thormbosis are coronary spasm, idiophatic thrombocytopenic purpura, antithrombin III deficiency, cocaine abuse and diabetes [9-13]. In our patient, except for a history of tabacoo use, poorly controlled lipid profile and diabetes, no other possible risk factors were identified. As regards the patient's diabetes, the diagnosis was established for the first time during STEMI hospitalization, which is observed frequently in patients presenting with acute myocardial infarction [14].

\section{Conclusions}

"The deadly double infarct syndrome", as the term suggests, is usually associated with patients' fatality. Therefore, emer- 
gent PCI leading to rapid restoration of coronary blood flow is paramount for their survival. As described above, in the era of pre-hospital ECG teletransmission, early performed PCI with excellent short-term clinical outcomes is possible. The case report ilustrates that in this unique group of patients, a quote "time is muscle" gains a particular importance or should even be paraphrased as "time is life". From this perspective, prehospital ECG teletransmission plays a particularly crucial role in fast initiation of guidelines-based treatment and patients' survival.

\section{Conflict of Interest}

None.

\section{Financial Support}

This research received no specific grant from any funding agency in the public, commercial or not-for-profit sectors.

\section{References}

1. Goldstein JA, Demetriou D, Grines CL, Pica M, Shoukfeh M, O'Neill WW. Multiple complex coronary plaques in patients with acute myocardial infarction. N Engl J Med. 2000;343(13):915-922.

2. Pollak PM, Parikh SV, Kizilgul M, Keeley EC. Multiple culprit arteries in patients with ST segment elevation myocardial infarction referred for primary percutaneous coronary intervention. Am J Cardiol. 2009;104(5):619623.

3. Hakim J, Mehta A. The deadly double infarct syndrome treated by two-vessel primary angioplasty and stenting. J Invasive Cardiol. 2000;12(1):29-33.

4. Fukaya H, Oikawa J, Hirasawa S, Shimohama T, Tojo T, Niwano S, Izumi T. Acute myocardial infarction involving double vessel total occlusion of the left anterior descending and left circumflex arteries: A case report. J Cardiol Cases. 2011;4:e1-e4.

5. Burke A, Virmani R. Significance of multiple coronary artery thrombi. A consequence of diffuse atherosclerotic disease? Ital Heart J. 2000;1(12):832-834.

6. Maehara A, Mintz GS, Bui AB, Walter OR, Castagna MT, Canos D, Pichard AD, et al. Morphologic and angiographic features of coronary plaque rupture detected by intravascular ultrasound. J Am Coll Cardiol. 2002;40(5):904910.

7. Kurisu S, Inoue I, Kawagoe T, Ishihara M, Shimatani Y, Mitsuba N, Hata T, et al. Aspiration thrombectomy for acute myocardial infarction resulting from the sequential occlusions of two major coronary arteries in a short time. Intern Med. 2004;43(12):1166-1170.

8. Ribeiro H, Ferreira C, Batista A, Magalhaes P, Margato R, Carvalho S, Ferreira A, et al. [Sequential double vessel myocardial infarction]. Rev Port Cardiol. 2011;30(12):925-927.

9. Suzuki N, Hiasa Y, Miyazaki S, Tomokane T, Ogura R, Miyajima H, Ohara Y, et al. Acute myocardial infarction caused by simultaneous occlusion of the right coronary artery and the left anterior descending coronary artery probably due to coronary spasm: a case report. J Cardiol. 2005;45(5):213-217.

10. Yagmur J, Cansel M, Acikgoz N, Yagmur M, Eyupkoca F, Ermis N, Akturk E. Multivessel coronary thrombosis in a patient with idiopathic thrombocytopenic purpura. Tex Heart Inst J. 2012;39(6):881-883.

11. Tu CM, Hsueg CH, Chu KM, Cheng SM, Tsao TP. Simultaneous thromboses of double coronary arteries in a young male with antithrombin III deficiency. Am J Emerg Med. 2009;27(9):1169 e1163-1166.

12. Meltser H, Bhakta D, Kalaria V. Multivessel coronary thrombosis secondary to cocaine use successfully treated with multivessel primary angioplasty. Int J Cardiovasc Intervent. 2004;6(1):39-42.

13. Ikeda Y, Fujinaga H, Niki T. Successful percutanous coronary intervention for acute myocardial infarction caused by simultaneous occlusion of two major coronary arteries in patients with diabetes mellitus. A report of two cases. Acta Cardiol. 2005;60(2):225-228.

14. Arnold SV, Lipska KJ, Li Y, McGuire DK, Goyal A, Spertus JA, Kosiborod M. Prevalence of glucose abnormalities among patients presenting with an acute myocardial infarction. Am Heart J. 2014;168(4):466-470 e461. 